\title{
Rúbricas como estrategia de evaluación en entornos TICS Rubrics as an evaluation strategy in ICT environments
}

\author{
V. Torres-Sanz ${ }^{1}$, Piedad Garrido ${ }^{1}$, Julio A. Sanguesa ${ }^{1}$, Francisco J. Martinez ${ }^{1}$, J. Tramullas ${ }^{2}$ \\ vtorres@unizar.es,piedad@unizar.es,jsanguesa@unizar.es,f.martinez@unizar.es, tramullas@unizar.es \\ ${ }^{1}$ Departamento de Informática e Ingeniería de \\ Sistemas \\ Universidad de Zaragoza \\ Teruel, España \\ ${ }^{2}$ Departamento de Ciencias de la Documentación e \\ $\mathrm{H}^{\mathrm{a}}$ de la Ciencia \\ Universidad de Zaragoza \\ Zaragoza, España
}

\begin{abstract}
Resumen- La enseñanza de las asignaturas de Tecnologías de la Información y Comunicaciones (TICs) enmarcadas en titulaciones de Ciencias Sociales, es de gran importancia y elevada complejidad. Por eso se requiere una metodología de trabajo adecuada, que facilite el correcto aprendizaje por parte de los alumnos. Sin embargo aún es bastante habitual utilizar metodologías de enseñanza basadas en la escuela tradicional, en donde se abusa de clases magistrales, se califica a los alumnos utilizando una única prueba de evaluación final y en caso de realizar algún ejercicio práctico, la única retroalimentación que reciben los alumnos es su calificación numérica, a veces incluso después de realizar los exámenes. En este trabajo se expondrá el uso de una metodología de trabajo innovadora, adaptada al Espacio Europeo de Educación Superior (EEES), basada en la utilización del Aprendizaje Basado en Proyectos Colaborativos (ABPC) en la que además se ha utilizado como estrategia evaluadora la utilización de rúbricas. Los resultados obtenidos son muy esperanzadores, consiguiendo una participación activa por parte de los alumnos, una reducción del absentismo y los problemas derivados de la disciplina, permitiendo atender a la diversidad y heterogeneidad del alumnado, y lo que es más importante, fomentan el aprendizaje de todo el aula, lo que es altamente satisfactorio e inusual para la actividad académica en educación superior.
\end{abstract}

Palabras clave: ABPC, evaluación, EEES, rúbrica, competencia

\begin{abstract}
Information and Communications Technology (ICT) teaching subjects, framed in Social Sciences degrees, is of great importance and high complexity. That is why a proper working methodology is required, which facilitates the correct learning on the part of the students. However, it is still quite common to use teaching methods based on the traditional school, where lectures are abused, students are graded using a single final exam and in the case of some practical exercise, the only feedback students received is their numerical rating, sometimes even after the exams. This work will show the experience of using an innovative work methodology, adapted to the European Higher Education Area (EHEA), and based on the use of Collaborative Project-Based Learning (CPBL) together with the innovative strategy of using rubrics. The results obtained are very encouraging, getting an active participation by the students, absenteeism reduction and problems arising from discipline, allowing to attend to the diversity and heterogeneity of the students, and what is more important, to get the whole classroom learning, which is highly satisfactory and unusual for academic activity in higher education
\end{abstract}

Keywords: CPBL, evaluation, EHEA, rubric, competency

\section{INTRODUCCIÓN}

Desde la perspectiva del modelo tradicional de enseñanza, la evaluación es la última etapa del proceso didáctico, etapa en la que se realiza un proceso de selección en base a un conjunto de objetivos y criterios establecidos. El resultado de esta comparación es utilizado para valorar el trabajo de los alumnos, ya sea de manera positiva o negativa. Con esta situación lo que se provoca, es un ritmo forzado de trabajo y una formación excesivamente docta, técnica y académica, en la que el procedimiento de evaluación constituye un proceso distintivo del aprendizaje. Por un lado se encuentran el resto de las funciones docentes, (se enseña, se practica, se demuestra...) con un objetivo claramente didáctico, mientras que por el otro se evalúa, como un proceso aislado y sin carácter formativo. De esta manera los procesos de evaluación parecen convertirse en procesos con carácter accidental y no deseable, en los que el docente rehúsa la interacción y la formación del alumno en base al establecimiento de una calificación numérica.

Sin embargo la sociedad busca unos resultados (a poder ser numéricos), baremados en un sistema de calificaciones, que permitan valorar el éxito o fracaso de un alumno en su aprendizaje. Lo cual nos hace pensar si este es un método válido para evaluar la capacidad de aprendizaje o la valía que tendrá el alumno para prosperar en su futuro laboral. Si en vez de plantear qué es la evaluación nos centramos en qué debería de significar, tal vez una definición más correcta sea la siguiente (Rosales, 2011):

“La evaluación constituye una reflexión crítica sobre todos los momentos y factores que intervienen en el proceso didáctico a fin de determinar cuáles pueden ser, están siendo o han sido los resultados del mismo."

El Joint Committee on Standards for Educational Evaluation establece las siguientes cuatro normas a la hora de evaluar (Vargas, 2004):

- Útil: proporcionar información acerca de virtudes y defectos del trabajo, así como mejoras a realizar

- Factible: utilizar procedimientos evaluativos que sean fácilmente aplicable y entendibles

- Ética: protección de las partes implicadas y la honradez de los resultados 
- Exacta: describir la evolución y contexto del trabajo, revelando virtudes y defectos. Estar libre de influencias y proporcionar conclusiones

Teniendo en cuenta el marco del Espacio Europeo de Educación Superior (EEES), el concepto de evaluación adquiere una importancia mayor aún en el proceso de aprendizaje de los alumnos, ya que el examen tradicional es incapaz, por sí mismo y sin otros instrumentos de evaluación, de dar respuesta a las necesidades del modelo de la Convergencia Europea (Lirola, 2008)

Además, como comenta el psicólogo Jean Piaget en su obra Psicología y Pedagogía "Es importante poner en funcionamiento todos los medios para hacer progresar las técnicas de evaluación cualitativa y cuantitativa que permitan una verificación sistemática de los resultados obtenidos, ya que esta verificación debe facilitar la elaboración de planes ulteriores" (Piaget, 1969).

El objetivo principal de este trabajo es mostrar una nueva metodología de trabajo, en la que se combina el Aprendizaje Basado en Proyectos Colaborativos (ABPC) junto a una nueva estrategia a la hora de evaluar. Esta estrategia de evaluación está basada en la incorporación de rúbricas de evaluación, orientadas a materias TIC impartidas en entornos universitarios. El desarrollo de esta estrategia nos ha permitido encontrar una metodología de trabajo que permite ya no sólo evaluar el trabajo realizado por el alumno, sino el de evaluar: (I) las competencias adquiridas en su proceso de aprendizaje, (II) su capacidad de responder a demandas complejas, (III) proporcionar una retroalimentación que favorezca su aprendizaje significativo y (IV) el desarrollo de las competencias específicas y trasversales de cada titulación, teniendo en cuenta los principios del proyecto "Definición y Selección de Competencias, (DeSeCo), de la Organización para la Cooperación y el Desarrollo Económicos (OCDE)” (Bolívar, 2008).

La rúbrica es un instrumento de evaluación utilizado en numerosos ámbitos. Por ejemplo (Merino, 2011) la pone en práctica en la titulación de Grado de Educación Infantil como una estrategia para fomentar el trabajo en grupo. Para ello utilizó rúbricas analíticas para evaluar la adquisición de competencias por parte de su alumnado. En (Figueira, 2013) se pone en práctica la utilización de rúbricas en el Grado de Educación como instrumento de autoevaluación orientada al aprendizaje. En este caso en la rúbrica utilizada se ponía una nota numérica en base a una ponderada. En (Torres, 2011) aplican rúbricas analíticas en docencia on-line dentro del Master Universitario en Sostenibilidad y Responsabilidad Social Corporativa. En (Sanz, 2014) se fomenta la utilización de rúbricas analíticas en el Grado en Pedagogía, para evaluar la adquisición de competencias por parte de sus alumnos, utilizando la herramienta Rubistar. En (Rodríguez Gallego, 2014) se propone la utilización de la rúbrica holística. En este caso, la estructura de la rúbrica fue consensuada con los alumnos del Grado de Primaria para proponer una rúbrica que se adaptara mejor a las necesidades de los alumnos. En (Cano García, 2011) se apuesta por la utilización de rúbricas holísticas debido a que, en su opinión, ofrece una mayor fortaleza en la descripción y evaluación de competencias. Por eso propone su utilización como estrategia de evaluación dentro del EEES.
Si se realiza un pequeño análisis de los diferentes estudios expuestos, se observan las siguientes características:

1. Las investigaciones en las que se utilizan rúbricas suelen entablarse en ámbitos de ciencias sociales

2. Unos investigadores se decantan por un tipo de rúbrica (sea analítica u holística) descartando la otra

3. Suelen utilizar una estructura fija, es decir, la valoración de la adquisición de competencias por parte del profesor utiliza frases preestablecidas para todos los alumnos, por lo que no se personaliza en base al trabajo y las necesidades de cada estudiante

En este trabajo expondremos nuestra experiencia utilizando las rúbricas en un ambiente distinto y novedoso, la Ingeniería. Además, propondremos un nuevo tipo de rúbrica, que, en nuestra opinión, contiene los beneficios de ambas (analíticas y holísticas). Esta rúbrica huye de frases preestablecidas para todas las evaluaciones, siendo totalmente personalizable en base al trabajo y necesidades del alumno, y haciendo hincapié en aquellos factores en los que el alumno debe reforzar en su aprendizaje en cada una de las competencias que debe de adquirir.

Esta contribución está organizada de la siguiente manera: en la sección II se presenta el contexto de nuestra investigación en la que se trata de adaptar estrategias educativas de éxito al ámbito de la educación superior en el contexto de la Ingeniería. Esta sección contiene la necesidad de realización (Sección II-A) y el propósito de la investigación (Sección II-B). En la sección III se comentan los beneficios que tiene la utilización de rúbricas en la enseñanza (Sección III-A), además se describe la metodología utilizada (Sección III-B) y la situación del aula en la que se realizó. En la Sección IV se exponen los resultados obtenidos en nuestra investigación, y por último en la Sección $\mathrm{V}$ se comentan las conclusiones más importantes obtenidas.

\section{CONTEXTO}

\section{A. Necesidad de realización}

Los docentes de Ingeniería suelen utilizar metodologías de enseñanza basadas en la escuela tradicional. En este tipo de enfoque disciplinar el profesor es el centro del desarrollo de la clase, la cual se desarrolla mediante la utilización de clases magistrales. El alumno es un elemento pasivo, que se dedica a absorber los conocimientos expuestos por el docente, conocimientos que son expresados de manera muy académica. El proceso de evaluación va dirigido al resultado sin preocuparse del proceso de aprendizaje.

Teniendo en cuenta el marco del EEES, los docentes debemos paulatinamente ir cambiando el medio de transmisión de los conocimientos (Patier, 2008), permitiendo a los alumnos que puedan trabajar de forma autónoma. Se pretende transformar al profesor de un depositario del saber a un gestor del proceso de aprendizaje, y trabajar buscando objetivos basados en competencias (saber hacer) y no únicamente en conocimientos disciplinarios (saber) (Lirola, 2008). En este marco, los procesos de evaluación cobran mayor importancia.

Desde la Escuela Universitaria Politécnica de Teruel (EUPT), se lleva más de quince años trabajando con una nueva metodología de trabajo en la disciplina de Bases de 
Datos (BD), en las que se está utilizando como metodología de enseñanza el Aprendizaje Basado en Proyectos Colaborativos (ABPC), una metodología que combina el Aprendizaje Basado en Proyectos con el Aprendizaje Colaborativo (AC). Los resultados obtenidos con esta metodología son bastante positivos y esperanzadores.

Sin embargo se considera que esta metodología aún era susceptible de mejora. Por lo que se ha decidido incorporar a la metodología ABPC un sistema de evaluación basado en rúbricas, con el objetivo de proporcionar una mayor retroalimentación al alumno y favorecer la adquisición de competencias. No hay que olvidar que la evaluación es una oportunidad para promover el aprendizaje, sin embargo en muchos casos sólo es utilizada por muchos profesores para calificar de manera numérica la calidad del trabajo realizado por el estudiante.

Esta investigación educativa se ha llevado a cabo, durante este curso 2016-2017, con un grupo piloto, en el recién implantado Máster en Consultoría de Información y Comunicación (MCIC). Estos estudios son ofrecidos por la Facultad de Filosofía y Letras de la Universidad de Zaragoza, es un máster con orientación profesional que se ofrece en la modalidad semipresencial, y con alumnos de variada procedencia (ingeniería, periodismo, documentación, etc.). Los alumnos se encuentran, en general, en un rango de edad de entre 25 y 50 años.

En concreto, doce créditos del máster son asignaturas relacionadas con las TICs, impartidas por profesores del Departamento de Informática e Ingeniería de Sistemas (DIIS). Según la opinión del alumnado, a pesar de tratarse de un máster profesionalizante, el profesorado sigue abusando de un modelo de enseñanza tradicional basado en clases magistrales. También se les va a seguir calificando mediante la realización de una única prueba, obviando funciones básicas de la evaluación como lo son la de control, la de mejora de los procesos, la de autorregulación (Sallán, 2009) y la de retroalimentación, y sin tener en cuenta los beneficios de una educación inclusiva.

Dicho grupo piloto está formado por los alumnos de la asignatura obligatoria, de primer cuatrimestre, Tecnologías de la Información y Comunicación (TIC), cuyo contenido consta de los siguientes bloques: (i) Herramientas avanzadas de gestión de contenidos (CMS) recursos (ERP) y clientes (CRM), (ii) Metadatos, ontologías, web semántica y visualización de la información, (iii) Herramientas avanzadas de diseño gráfico y multimedia y realización audiovisual, (iv) Preservación y continuidad digital y, (v) Minería de datos y minería de texto.

\section{B. Propósito de la investigación}

El concepto de evaluación en la Educación Superior está cambiando desde un modelo centrado en el profesor o de «evaluación del aprendizaje» hacia otro modelo centrado en el alumno o de «evaluación para el aprendizaje» (Berrocoso, 2014).

Las rúbricas se han mostrado como un instrumento de éxito para convertir los procesos de evaluación en procesos de aprendizaje. Esto se ha visto refrendado en numerosas investigaciones, como por ejemplo las realizadas por (Berrocoso, 2014) que concluye que puntuar utilizando rúbricas es más fiable que evaluar sin ellas, o (Torres y
Perrera, 2011) y (Gordillo, 2006), que proponen la utilización de un tipo distinto de rúbrica o (Cabré et al., 2016) que utilizaron las rúbricas como mecanismo de evaluación, con una gran acogida tanto por parte de los profesores como por parte de los alumnos.

Puesto que el estudio de asignaturas TICs en entornos ajenos a Ingenierías Informáticas suele ser problemático y plantea serias dificultades a los alumnos, se decidió hacer un grupo piloto en el máster de enseñanza semipresencial MCIC, para tratar de cambiar la estrategia docente de una enseñanza tradicional a una adaptada al EEES, mediante la utilización del ABPC y utilizando las rúbricas como estrategia formativoevaluadora.

\section{DESCRIPCIÓN}

Se presentará en este epígrafe la sistemática seguida y los medios empleados.

\section{A. Rúbrica}

Aunque existen varios tipos de rúbricas, generalmente suelen describirse dos: las analíticas y las holísticas. En las primeras se desglosan los criterios a evaluar en varios niveles de ejecución, mientras que las segundas evalúan el trabajo en su totalidad, de manera descriptiva. Sin embargo, ninguno de estos dos modelos se adaptaba con exactitud a los objetivos buscados, por lo que se creó un nuevo tipo de rúbrica, que permitiese evaluar de una manera numérica la calidad del trabajo con mayor precisión, facilitase a los alumnos afrontar las actividades, y proporcionara un mayor nivel de retroalimentación, ajustándola más a las necesidades de evaluación de entornos TICs.

Las características generales de la rúbrica utilizada (Figura 1) son las siguientes:

- Claridad: el alumno sabe de antemano las características de la actividad, y se permite que gestione de manera autónoma cómo la va a afrontar

- Detallada: permite que sepa exactamente qué tiene que hacer y no realice cosas que no se piden

- Seguridad: el alumno conoce de antemano los criterios de evaluación y afronta la actividad con más seguridad

- Exactitud: permite retornar al alumno la calidad de su trabajo de manera exacta y precisa, ofreciéndole la nota de su actividad

- Evaluación: permite clasificar la evaluación según la adquisición de competencias, además de proporcionar un sistema de evaluación fiable

- Individualizado: ofrece información detallada sobre cómo está progresando el proceso de aprendizaje de manera individualizada

- Retroalimentación: proporciona un mayor nivel de retroalimentación a los alumnos, lo que favorece el aprendizaje del alumno 


\begin{tabular}{|c|c|c|c|}
\hline \multicolumn{4}{|c|}{ Tarea 2. Herramientas Avanzadas de Gestión } \\
\hline & Competencia (Nota) & Comentario & Nota \\
\hline \multirow{3}{*}{ 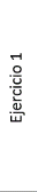 } & $\begin{array}{l}\text { Comprensión y aplicación de } \\
\text { conceptos }(2,5)\end{array}$ & & \\
\hline & Análisis Critico $(1,25)$ & & \\
\hline & $\begin{array}{l}\text { Presentación y exposición } \\
\text { del trabajo(1,25) }\end{array}$ & & \\
\hline \multirow{3}{*}{ 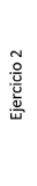 } & $\begin{array}{l}\text { Búsqueda de información } \\
\qquad(2,5)\end{array}$ & & \\
\hline & $\begin{array}{l}\text { Elaboración del contenido y } \\
\text { creatividad }(1,25)\end{array}$ & & \\
\hline & $\begin{array}{l}\text { Presentación y exposición } \\
\text { del trabajo(1,25) }\end{array}$ & & \\
\hline \multicolumn{4}{|c|}{ Nota final } \\
\hline
\end{tabular}

Figura 1 Ejemplo de rúbrica utilizada

\section{B. Metodología adoptada}

El primer paso es la creación de la actividad a evaluar, basada en la metodología ABPC. Una vez publicada, se procede al diseño de la rúbrica. Para ello se han seguido los siguientes pasos:

1. Considerar el contexto del proyecto o tarea a realizar, así como las competencias con las que se pretende trabajar

2. Describir los atributos o competencias y agruparlos en la rúbrica (resultados de aprendizaje esperados)

3. Diseñar la escala de valoración de cada atributo o competencia a valorar

4. Revisión y validación de la rúbrica para comprobar que cumple con los objetivos que se pretende alcanzar

Una vez creadas ambas, la actividad y la rúbrica, la metodología seguida ha sido la siguiente:

1. Se ha proporcionado a los alumnos el enunciado de la actividad a realizar.

2. Junto con el enunciado se ha entregado la rúbrica de evaluación de la actividad y se ha comentado en detalle los aspectos más importantes de la misma.

3. Los alumnos realizan y entregan la actividad.

4. Una vez corregida la actividad, se rellena la rúbrica de manera individualizada.

5. Se entrega a los alumnos la rúbrica con las particularidades de corrección. Se ha valorado, de manera general, cada competencia incluyendo los defectos, así como posibles mejoras.

Las características del aula en la que se puso en práctica esta metodología son un tanto particulares, puesto que estamos hablando de un Máster en Consultoría de Información y Comunicación en el que participaban veinticinco alumnos de diversa procedencia. En la asignatura cursada en el primer cuatrimestre, Tecnologías de la Información y Comunicación, se siguió una metodología de enseñanza tradicional. Los resultados obtenidos fueron bastante decepcionantes, con un elevado grado de absentismo, una gran desmotivación y desinterés por parte del alumnado, hasta el punto que llegaron a amotinarse, negándose a seguir cursando dicha asignatura en esas condiciones, y solicitando, al coordinador del máster, que se cambiase la orientación de la misma, puesto que consideraban que lo que se estaba impartiendo carecía de interés para su formación y dudaban incluso, de que tuviera alguna utilidad para su futuro como consultores.

La asignatura TIC impartida por cinco profesores, especialistas cada uno de ellos en un bloque de su contenido, propusieron actividades separadas de evaluación y, al recibir la queja del coordinador, adaptaron el contenido y el nivel de exigencia a las competencias del máster, pero eso no fue suficiente, hasta que llegó la clase relativa al bloque de "Herramientas avanzadas para la gestión de contenidos, recursos y clientes”, donde se aplicaron ambas metodologías (APBC + rúbricas), que aunque en la Web oficial del máster aparece en primer lugar, este curso académico fue el tercer bloque en impartirse.

\section{Resultados}

Antes de proceder a realizar un análisis de los resultados obtenidos, pensamos que es interesante hacer una recopilación de los números que estamos barajando. De un número inicial de veinticinco alumnos matriculados en el máster, a lo largo del primer cuatrimestre se dieron de baja cinco, por diversas causas: personales, médicas y por no cumplir, el máster, las expectativas deseadas. $\mathrm{Y}$ de los veinte restantes, dieciocho se matricularon en la asignatura TIC. Tras impartirse las dos primeras sesiones presenciales del máster, prácticamente el $70 \%$ de la clase decidieron no realizar los entregables de dichos bloques e incluso abandonar la materia. Después de la sesión presencial del tercer bloque, en el que se le planteó al alumnado la realización de una actividad de APBC acompañada de su rúbrica de evaluación, junto al esfuerzo realizado por el profesorado de la materia de readaptación de contenidos, la actitud cambió y, en los dos restantes bloques, fueron más receptivos y optaron por darle una nueva oportunidad a la materia. Siendo los resultados de la evaluación continua más la prueba final, los siguientes:

Tabla 1 Resultados de la primera convocatoria

\begin{tabular}{|c|c|c|}
\hline NP & APROBADOS & NOTABLES \\
\hline 3 & 6 & 9 \\
\hline
\end{tabular}

Si comparamos los datos de principios de cuatrimestre con los resultados finales, se observa que sólo con ese pequeño detalle de introducción de nuevas metodologías en el aula tanto de aprendizaje como de evaluación, se redujo en aproximadamente un 56\% la actitud de negación absoluta hacia la realización de las tareas y la tasa de abandono a la que nos enfrentábamos.

Además de los resultados cuantitativos comentados con anterioridad, se percibieron las siguientes mejoras en los alumnos:

- Reducción de la ansiedad: al aumentarse la trasparencia y clarificar los criterios de evaluación se percibió un aumento de los niveles de confianza por parte de los alumnos. Estos se enfrentaban a las actividades con más seguridad al conocer qué se esperaba de ellos y cómo se les iba a evaluar 
- Retroalimentación: los alumnos se mostraron encantados con la retroalimentación que les aportaba las rúbricas a sus actividades. De esta manera además les permitía reflexionar sobre la calidad de su trabajo de manera más objetiva

- Mejora de la eficacia y la autorregulación: varios alumnos mencionaron que al conocer las características del proceso de evaluación les permitía ser más eficaces y regularse mejor a la hora de realizar las actividades. Algo que en un máster semipresencial es de gran importancia

- Mayor motivación: debido a la metodología utilizada, los alumnos afrontaron las actividades con mayor motivación e interés

\section{CONCLUSIONES}

El proceso de evaluación no tiene porqué ser un proceso distintivo del aprendizaje, hay que tener en cuenta que la evaluación es una oportunidad para promover el aprendizaje. En este trabajo proponemos una forma distinta de trabajar, que es la utilización del ABPC junto a la incorporación de un nuevo tipo de rúbrica como estrategia evaluadora. Este tipo de rúbrica contiene los beneficios de las analíticas y las holísticas, además de ser totalmente personalizable en función de las necesidades del alumno. Esta estrategia, adaptada al EEES, ha sido probada en una titulación oficial de enseñanza tradicional con unos resultados excepcionales, tanto en la parte académica como en la actitud del alumnado en la asignatura.

Este cambio radical mostrado en el comportamiento del grupo piloto, compuesto por un grupo piloto inicial de veinticinco alumnos del máster MCIC de la UZ, nos anima a poner en marcha la experiencia en asignaturas de titulaciones de grado, especialmente en ingeniería, aunque suponga una carga de trabajo más elevada para el profesorado, puesto que ya que el contenido no les es tan ajeno, esperamos poder mejorar otros aspectos en este tipo de alumnado, como son: la falta de motivación e iniciativa, la autorregulación en su tiempo de estudio personal, y fomentar el respeto no sólo hacia el profesorado sino también al resto de compañeros, cumpliendo por ejemplo con los plazos de entrega, valores claves de la educación y que escasean hoy en día en este tipo de formación.

\section{AGRADECIMIENTOS}

Este trabajo ha recibido apoyo financiero de la convocatoria de Proyectos de Innovación Docente 2016/17 de Universidad de Zaragoza, dentro del Programa de Incentivación de la Innovación Docente (PIIDUZ_16_380).

\section{REFERENCIAS}

Berrocoso, J. V. (2014). El uso de e-rúbricas para la evaluación de competencias en estudiantes universitarios. Estudio sobre fiabilidad del instrumento. Revista de Docencia Universitaria, 49-79.

Bolívar, A. P. (2008). El Proyecto DeSeCo sobre la definición y selección de competencias clave. Introducción a la edición española. Las competencias clave para el bienestar personal, social y económico, 1-13.

Cabré, R. B.-P.-P. (2016). Construcción y validación de Rúbricas para evaluar las prácticas de Diseños de Investigación. Revista del Congrés Internacional de Docència Universitària i Innovació (CIDUI).

Cano García, E. (2011). La evaluación por competencias en la educación superior. Profesorado. Revista de Curriculum y Formación del Profesorado .

Figueira, M. E. (2013). La rúbrica como instrumento para la autoevaluación: un estudio piloto. REDU: Revista de Docencia Universitaria.

Gordillo, J. J. (2006). La rúbrica como instrumento pedagógico para la tutorización y evaluación de los aprendizajes en el foro online en educación superior. Revista de Medios y Educación, 141-149.

Lirola, M. M. (2008). La evaluación en el marco del EEES: El uso del portfolio en Filología Inglesa. Revista de Docencia Universitaria, 1(2), 15.

Merino, E. C. (2011). Una propuesta de evaluación para el trabajo en grupo mediante Rúbrica. Escuela abierta, 6781.

Patier, C. C. (2008). La evaluación de la docencia ante el reto del Espacio Europeo de Educación Superior (EEES). Educación $X X 1,20$.

Piaget, J. (1969). Psicología y pedagogía. Barcelona: Ariel.

Rodríguez Gallego, M. R. (2014). Evidenciar competencias con rúbricas de evaluación. Escuela Abierta, 117-134.

Rosales, C. (2011). Criterios para una evaluación formativa. Narcea.

Sallán, J. G. (2009). Usos y abusos en la evaluación: la evaluación como autorregulación. En S. G. Educación, Usos y abusos en la evaluación: la evaluación como autorregulación (págs. 11-44). Subdirección General de Información y Publicaciones. Ministerio de Educación.

Santiago, K. \&. (2014). Evaluación educativa. Alianza Editorial.

Sanz, M. P. (2014). La evaluación de competencias en Educación Superior mediante rúbricas: un caso práctico. Revista electrónica interuniversitaria de formación del profesorado, 87-106.

Torres, M. J. (2011). Aplicación de las rúbricas de evaluación en la docencia on-line. Jornada sobre Aprendizaje Cooperativo SPIEU, USE-UJI. Castellón.

Vargas, A. I. (2004). La evaluación educativa: concepto, períodos y modelos. Actualidades Investigativas en Educación, 4(2), 28. 